
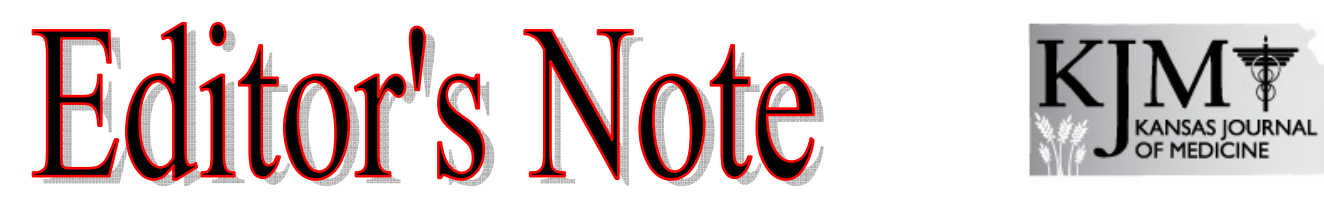

\title{
Breast Cancer Survivorship
}

Jon P. Schrage, M.D., M.P.A.

Editor, Kansas Journal of Medicine

University of Kansas School of Medicine-Wichita

Department of Internal Medicine

This issue of the Kansas Journal of Medicine (KJM) focuses on the subject of breast cancer survivorship. Breast cancer survivorship plays an integral role in the breast cancer care continuum. Survivorship care planning from the time of diagnosis allows health care professionals to provide a variety of services to women at all stages of breast cancer diagnosis and treatment. Survivorship care offers services from a multi-disciplinary medical team that includes primary care physicians, medical oncologists, endocrinologists, dietitians, clinical psychologists, and other health care professionals. Patients receive physical examinations, genetic counseling, cardiology assessments, diet, exercise programs, and fertility preservation. Two breast cancer survivorship centers have been created in Kansas; one in Kansas City and one in Wichita. These Centers seek to fill the unmet needs of breast cancer survivors by focusing on the physical and emotional needs of breast cancer survivors, family, and friends. The research studies presented in this issue report on multiple focus groups with breast cancer survivors and individuals involved in breast cancer care. The focus groups clearly indicated several psychosocial issues associated with diagnosis and treatment that need to be addressed to ensure an optimal outcome. As survivorship care is enhanced in Kansas, these studies point a clear direction for moving forward. 